Diabetologe 2010 - 6:253-254

DOI 10.1007/s11428-010-0585-8

Online publiziert: 11. Juni 2010

๑) Springer-Verlag 2010

\author{
J. Kruse ${ }^{1} \cdot$ B. Kulzer ${ }^{2}$ \\ ${ }^{1}$ Klinik für Psychosomatik und Psychotherapie, Justus-Liebig-Universität Gießen \\ 2 Diabetes-Zentrum Mergentheim, Forschungsinstitut Diabetes-Akademie \\ Bad Mergentheim (FIDAM), Bad Mergentheim
}

\title{
Diabetes und Depression
}

In den letzten Jahren wurde eine Vielzahl psychosomatischer Zusammenhänge im Bereich der Diabetologie entdeckt. Psychosozialer Stress sowie subklinische und klinische psychische Störungen beeinflussen gleichermaßen den Lebensstil, das Selbstbehandlungsverhalten, die Stoffwechseleinstellung, aber auch die langfristige Prognose und Mortalität des Diabetes erheblich.

Paradigmatisch wurde vor allem die Beziehung zwischen Diabetes und Depression untersucht, was sich auch in der rasanten Zunahme der internationalen Publikationen zu diesem Thema widerspiegelt. Als ein zentrales Ergebnis der aktuellen Studienlage kann betrachtet werden, dass Diabetespatienten sowohl mit subklinischer als auch klinischer Depression eine deutlich erhöhte Mortalitätsrate gegenüber diabeteserkrankten Patienten ohne depressive Symptomatik aufweisen. Marc Peyrot spricht in seinem Editorial in Diabetes Care von „Depression: a quiet killer by any name“.

Die Erforschung des Zusammenhangs zwischen Depression und Diabetes ist eine spannende Aufgabe, wofür auch der Umstand spricht, dass mit DIAMANT („Diabetes and Mental Aspects“) und DECODIA („Depression and cognition in diabetes. From neurobiological mechanisms to treatment strategies") zwei der sieben Forschungsverbünde des vom Bundesministerium für Bildung und Forschung (BMBF) geförderten „Kompetenznetzes Diabetes mellitus" sich mit dem Zusammenhang von Diabetes und Depression beschäftigen. Auch in einem Versorgungsforschungsprojekt der Bundesärztekammer wird diese Fragestellung intensiv bearbeitet.

\section{(7) Depressionen erschweren die Modifikation des Lebensstils und die Durchführung der Therapie im Alltag}

Die Assoziation Diabetes und Depression ist jedoch auch von unmittelbarer klinischer Relevanz, denn Depressionen erschweren die Modifikation des Lebensstils und die Durchführung der Therapie im Alltag. Gerade wegen der depressiven Problematik ist der Zugang zu diesen Patienten oft schwieriger und in der Praxis aus Zeitgründen oft limitiert. Für Patienten ist die Depression mit einer reduzierten Lebensqualität und oft einer schlechteren Blutzuckereinstellung und Folgekomplikationen des Diabetes verbunden. Menschen mit Diabetes sollte daher in der klinischen Praxis vermehrt Aufmerksamkeit geschenkt werden.

Eine aktuelle Studie der amerikanischen Arbeitsgruppe um Katon konnte erneut aufzeigen, dass die Kosten für die Behandlung von depressiven Diabetespatienten um ein Vielfaches höher liegen als bei Patienten ohne Depression. Allerdings konnte mit einem strukturierten Vorgehen („,stepped care approach") nicht nur eine deutliche 
Verbesserung der Depression, sondern auch eine Senkung der Behandlungskosten erreicht werden. Eine bessere Versorgung dieser Patienten könnte daher sowohl eine Verbesserung der Situation von Menschen mit einer Depression und Diabetes als auch gleichermaßen einen Beitrag zur Kostenreduktion bei einer großen Gruppe von Diabetespatienten leisten.

Voraussetzung dafür ist allerdings eine bessere Früherkennung und Therapie bei dieser Patientengruppe. In der Praxis werden noch immer weniger als $50 \%$ aller Menschen mit Diabetes und einer Depression diagnostiziert. In diesem Schwerpunktheft möchten wir Sie daher über die wichtigsten Zusammenhänge zum Thema Depression und Diabetes informieren und Ihnen die für die Praxis wichtigsten Fragen hinsichtlich der Erkennung und Behandlung depressiver Symptome und klinisch manifester Depressionen bei Menschen mit Diabetes beantworten.

Wir wünschen Ihnen viel Freude beim Lesen!

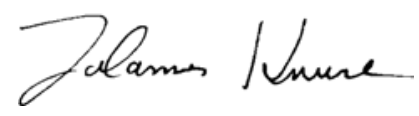

J. Kruse

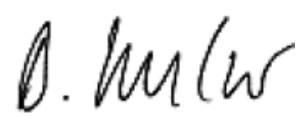

B. Kulzer

\section{Korrespondenzadressen}

\section{Prof. Dr. J. Kruse}

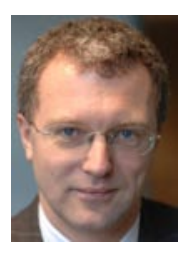

Klinik für Psychosomatik und Psychotherapie, JustusLiebig-Universität Gießen Friedrichstr. 33, 35392 Gießen johannes.kruse@psycho. med.uni-giessen.de

PD Dr. phil. Dipl.-Psych. B. Kulzer

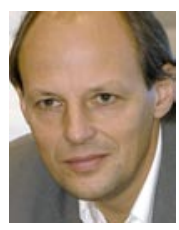

Diabetes-Zentrum

Mergentheim,

Forschungsinstitut

Diabetes-Akademie

Bad Mergentheim (FIDAM)

Theodor-Klotzbücher-Str. 12,

97980 Bad Mergentheim

kulzer@diabetes-zentrum.de

\section{Neu identifizierte Genloci fördern die Entstehung von Diabetes}

Das internationale Forschungskonsortium MAGIC (Meta-Analyses of Glucose and Insulin-related traits Consortium) hat in einer Meta-Analyse neue Genbereiche identifiziert, die den Nüchternblutzucker beeinflussen. Einige dieser Gen-Loci sind direkt mit einem erhöhten Diabetesrisiko assoziiert.

Auf Grund dieser Daten kann nun gezielter erforscht werden, welche Stoffwechselmechanismen zu einem erhöhten Nüchternblutzucker führen und wie die Gene die Diabetesentstehung beeinflussen. Die Ergebnisse des Konsortiums, an dem auch Forscher des Deutschen Instituts für Ernährungsforschung beteiligt sind, wurden nun in Nature Genetics veröffentlicht.

Die Wissenschaftler analysierten etwa 2,5 Mio. Single- Nukleotid-Polymorphismen (SNPs), die im Zusammenhang mit dem Nüchternblutzuckerspiegel, dem Nüchterninsulinspiegel, der Insulinresistenz und der Betazell-Funktion stehen sollen. Die SNPs waren im Rahmen von 21 genomweiten Assoziationsstudien identifiziert worden, die Daten von 46.186 nicht an Diabetes erkrankten Studienteilnehmern europäischer Abstammung einschlossen.

Literatur: Dupuis J, Langenberg C, Prokopenko l et al (2010) New genetic loci implicated in fasting glucose homeostasis and their impact on type 2 diabetes risk. Nature Genetics 42: 105-116

Quelle:

Deutsches Instituts für Ernährungsforschung Potsdam-Rehbrücke, www.dife.de

\section{Hormonerkrankungen durch Weichmacher}

Weichmacher für Kunststoffe - die sogenannten Phthalate - kommen immer noch in hohen Konzentrationen in vielen Medizinprodukten und Medikamenten vor, obwohl es Hinweise auf schädliche Wirkungen beim Menschen gibt.

Phthalate werden in großen Mengen industriell erzeugt und für die Fertigung von Kunststoffen wie PVC oder synthetischem Gummi verwendet. Bei bestimmten Phthalaten gibt es Hinweise auf schädliche Wirkungen beim Menschen. Diese sind von der EU in Kosmetika oder Kinderspielzeug verboten. In Lebensmittelverpackungen sind inzwischen geringere Grenzwerte als früher vorgeschrieben. In vielen Medizinprodukten wie Blutbeuteln, Infusionsbeuteln, Schläuchen oder Kathetern sind jedoch nach wie vor hohe Konzentrationen enthalten. Sie beeinflussen das menschliche Hormonsystem und gehören deshalb zu den Endokrinen Disruptoren.

Phthalate können durch Kontakt mit Blut oder Infusionslösungen leicht aus dem Kunststoff herausgelöst werden. Nehmen Schwangere solche Präparate ein, kann dies bereits zu Schäden bei männlichen Föten führen: Phthalate stören die Synthese von Testosteron. Die Folge im Erwachsenenalter kann eine verminderte oder fehlende Fruchtbarkeit der betroffenen Männer sein.

Mehrere deutsche und US-amerikanische Studien haben bereits endokrin aktive Phthalate im Urin der Allgemeinbevölkerung nachgewiesen. Zudem zeigen aktuelle Studien, dass sich unterschiedliche Phthalate auch in geringerer Konzentration in ihrer schädigenden Wirkung addieren.

Auf Grund der potenziell schädigenden Wirkung sollten Kliniken auf Medizinprodukte umstellen, die kein DEHP oder DBP enthalten.

Seit dem 21. März 2010 müssen DEHPhaltige Medizinprodukte EU-weit gekennzeichnet werden.
Quelle: Deutsche Gesellschaft für Endokrinologie (DGE), www.endokrinologie.net 